\title{
Autistic-Like Traits in Laboratory Rodents Exposed to Phthalic Acid Esters During Early Development - an Animal Model of Autism?
}

\author{
Martina MOROVÁ ${ }^{1}$, Lucia KRŠKOVÁ ${ }^{1}$ \\ ${ }^{1}$ Department of Animal Physiology and Ethology, Faculty of Natural Sciences, Comenius \\ University in Bratislava, Bratislava, Slovak Republic
}

Received September 2, 2020

Accepted February 25, 2021

Epub Ahead of Print May 12, 2021

\begin{abstract}
Summary
Phthalates are chemical substances that are widely used to provide flexibility and durability to plastic materials. They leach from products in which they are mixed and reach living organisms. Results from experimental studies suggest that exposure to phthalates can have a negative impact on an individual's neuronal system and behavior. In this regard, exposure during early ontogenesis seems to be particularly dangerous due to the extensive growth and development of body structures and functions. Disruption during this critical time can result in alterations of behavior and the emergence of neurodevelopmental disorders, such as autism spectrum disorder (ASD). Various animal models have been used to elucidate the pathogenesis of this disease. They are fundamental for research, and although the translation of results to humans is difficult, new animal models are being developed. The aim of this review is to summarize laboratory rodent studies in which early developmental phthalate exposure resulted in brain alterations and autistic-like behavioral traits. We also discuss the possibility of using early developmental phthalate exposure in rodents to create a new animal model of autism.
\end{abstract}

\section{Key words}

Phthalates • Early-life exposure • Brain development • Behavior • Animal model

\section{Corresponding author}

M. Morová, Department of Animal Physiology and Ethology, Faculty of Natural Sciences, Comenius University in Bratislava, Mlynská dolina, Ilkovičova 6, 84215 Bratislava 4, Slovak Republic. E-mail: morova4@uniba.sk

\section{Introduction}

The diesters of 1,2-benzenedicarboxylic acid (phthalic acid) are commonly known as phthalates. Phthalates are man-made chemical substances that are presently widely used to provide flexibility and durability to plastic materials and that can also act as solvents. They can be found in a wide range of products used in daily life, such as vinyl flooring, shower curtains, raincoats, bath products, nail polish, perfumes, cosmetics, medications, and food storage containers (Schettler 2006, Diamanti-Kandarakis et al. 2009). As there is no covalent bond between phthalates and the plastic, in which they are mixed, phthalates may leach or outgas into their surroundings. They are widespread in the environment and the sources of exposure vary. They can enter our bodies parenterally (while delivering intravenous injections), but also through dermal contact (personal care products), inhalation (indoor air and dust contain phthalates that leach/outgas from various household products), and mostly food consumption (contaminated food) (Schettler 2006, Swan 2008, Mullerová et al. 2016).

Phthalates are usually quickly metabolized into monoester metabolites after they enter the body. Lowmolecular-weight phthalates are subsequently excreted and high-molecular-weight phthalates are further conjugated, and then excreted (Frederiksen et al. 2007). Their half-life is usually less than $24 \mathrm{~h}$ (Koch et al. 2005, Klein et al. 2016). Phthalate monoesters are used as biomarkers of exposure to both low-molecular and high- 
molecular-weight phthalates (Frederiksen et al. 2007). Phthalate metabolites are detected in bodily fluids including urine, saliva, amniotic fluid, or breast milk (Frederiksen et al. 2007). The concentration of these metabolites is usually estimated from urine samples (Swan 2008). The rate of exposure to phthalates is repetitive and relatively constant over time, and several authors have agreed that exposure assessment can be based on a single urine sample (Hoppin et al. 2002, Hauser et al. 2004).

Various studies have already demonstrated that phthalates have a negative impact on living organisms (Blystone et al. 2010, Boberg et al. 2011). These compounds have been studied in terms of their effects on susceptible subjects, including pregnant women, infants, and children (Factor-Litvak et al. 2014, Weng et al. 2017, Kolatorova et al. 2018, Tsai et al. 2018, Zhang et al. 2018).

Phthalates are endocrine disruptors, which means they can interfere with the function of natural blood-borne hormones (Diamanti-Kandarakis et al. 2009). Fetal exposure to phthalates can have negative effects on the function of the reproductive system of humans and animals (Swan et al. 2005, Hsieh et al. 2008, Repouskou et al. 2019). This impact has been studied intensively and has already been reviewed elsewhere (Martino-Andrade and Chahoud 2010, Zamkowska et al. 2018).

Phthalates can also have a negative impact on the developing brain and there has been discussion about a possible role of phthalates in the etiology of neurodevelopmental disorders, e.g. autism spectrum disorder (ASD) (Colborn 2004, Grandjean and Landrigan 2006).

Animal models have an important role in the field of science. Large numbers of animal models are currently being used to elucidate the causes behind various human disorders, including ASD (Olexová et al. 2012, Ergaz et al. 2016, Möhrle et al. 2020). ASD is characterized by impaired social interaction and social communication, as well as restricted and/or repetitive behaviors and interests (American Psychiatric Association 2013). Several methods are used to induce an autistic phenotype in animals, including the administration of exogeneous chemicals (Ergaz et al. 2016).

In this review, we will address the role of prenatal and early postnatal phthalate exposure in brain development and review rodent studies in which the impact of phthalates on the brain was examined. This aspect draws much attention and is increasingly being studied.

Next, we will discuss the presence of autisticlike traits in rodents that are often seen in studies investigating behavior after prenatal and early postnatal phthalate exposure.

Lastly, since rodents are usually used for the development of animal models of various disorders, we will consider the prospect of using rodents that were prenatally and early postnatally exposed to phthalates as a new potential animal model of ASD.

\section{Phthalates and their impact on brain development}

The brain is highly vulnerable and sensitive to exogenous factors at its early developing stage. This susceptibility originates from the fact that, during a short period of prenatal and early postnatal life, the brain develops from a strip of cells into a complex organ consisting of billions of precisely located, highly interconnected, and specialized cells (Grandjean and Landrigan 2006). Exposure to environmental chemicals during the brain growth spurt in the prenatal period has been suggested as a possible causal factor for impaired neurodevelopment and the emergence of neurodevelopmental and behavioral disorders, including ASD (Colborn 2004).

Only a limited number of studies have investigated the effect of phthalates on brain development. Therefore, the underlying mechanism, induced by prenatal and early postnatal phthalate exposure, that could lead to an autistic phenotype is not yet clear. Structural, functional, or molecular abnormalities in the hippocampus, amygdala, and prefrontal cortex are often mentioned. Pathomechanisms leading to these abnormalities were investigated in studies focusing solely on ASD, but also in some studies focusing on the role of phthalate exposure in brain development and ASD-linked changes. Below, we review these studies.

\section{Abnormalities in the hippocampus}

The hippocampus has for long been known to have an important role in spatial memory that enables spatial navigation (O'Keefe and Dostrovsky 1971). However, spatial memories are not the only ones supported by the hippocampus. It is also crucial for declarative memory, the ability to learn and remember everyday facts and events (reviewed in Cohen 2015). It was also found that the hippocampus is essential for social memory formation (Hitti and Siegelbaum 2014) 
and that it supports decision making and helps us to use memory in order to guide our behavior (Anderson et al. 2014, Cohen 2015).

Atypical hippocampal functioning was suggested as playing a role in the etiology of ASD (Ring et al. 2017), however, exact pathomechanisms are still to be elucidated. Synaptopathology and neuron loss are often mentioned.

Excitation and inhibition imbalances in synaptic transmission have been implicated in ASD (Lee et al. 2017). A decrease in the density of parvalbuminexpressing interneurons in the hippocampus was suggested as a potential mechanism for impaired social cognition in a study by Piskorowski et al. (2016). These interneurons provide an inhibitory output onto their target cells (Bartos and Elgueta 2012) and were found to be crucial for social memory (Deng et al. 2019). Furthermore, mutations in genes that are essential for synaptic function were found in ASD individuals (reviewed in detail in Guang et al. 2018).

Abnormalities in the function of synapses in the hippocampus are often seen in experimental studies on laboratory rodents investigating the role of phthalates in brain development. Li et al. (2013) administered di-nbutyl phthalate (DBP) to pregnant rat dams from gestational day six and throughout lactation. Pups of these dams demonstrated signs of synaptopathology in the hippocampus. Expression of synaptophysin (a protein important for the formation, maintenance, and plasticity of synapses) was decreased, and both the slope and amplitude of field excitatory postsynaptic potentials were reduced. Moreover, loss of synapses, decreased number of presynaptic vesicles, reduced postsynaptic density, and a broader synaptic gap were observed. These authors suggest that DBP exposure may induce synaptic loss and synaptic function failure, leading to cognitive impairment (Li et al. 2013). Early postnatal exposure of experimental rats to di(2-ethylhexyl)phthalate (DEHP) resulted in a decreased axonal innervation to the hippocampus (Smith et al. 2011) and interfered with normal synaptogenesis and connectivity in this region of the brain (Smith and Holahan 2014). Furthermore, hippocampal brain-derived neurotrophic factor (BDNF) mRNA expression was downregulated. BDNF is important for dendritic outgrowth and the formation of new synaptic connections and it was speculated that its downregulation could be one of the mechanisms by which phthalates affect the brain (Smith and Holahan 2014). Moreover, the role of BDNF was also acknowledged in the development of ASD (Qin et al.
2016). Dai et al. (2015) suggested another possible mechanism, analyzing the protein expressions for n-methyl-d-aspartate (NMDA) receptor subunits in the hippocampus after prenatal and early postnatal exposure to DEHP. NMDA receptors play a critical role in the establishment of synaptic connections and in the hippocampus, they are involved in long-term potentiation, a cellular model for learning and memory. Western blot analysis showed that the levels of receptor subunits were decreased in male brains and suggested that the development of the brain could be adversely affected (Dai et al. 2015). The role of NMDA receptors in ASD pathogenesis was also acknowledged (Sceniak et al. 2019). Moreover, an increase in synapsin-1 levels was seen in the hippocampus of both male and female rats after prenatal and early postnatal phthalate exposure (DeBartolo et al. 2016). Synapsins are important regulators of synaptic vesicle trafficking. Changes in their levels may skew excitatory/inhibitory synaptic transmission ratios (Gitler et al. 2004) and were implied in the pathogenesis of ASD (Chen et al. 2014).

Another possible pathomechanism was investigated in a study by Dong et al. (2018). Endoplasmic reticulum (ER) stress, oxidative stress, and apoptosis were studied in different regions of the autistic brain, including the hippocampus. These authors discovered that ER stress signals were activated, and that the occurrence of ER stress is possibly caused by oxidative stress and leads to apoptosis. It was suggested that these changes can play a role in the pathogenesis of autism (Dong et al. 2018).

The possibility of phthalate-induced apoptosis of hippocampal neurons was investigated in a study by $\mathrm{Li}$ et al. (2013). Rat pups of dams that were administered DBP from gestational day six and throughout lactation suffered cell apoptosis of the hippocampal neurons ( $\mathrm{Li}$ et al. 2013). Furthermore, hippocampal neuron loss and structural alternations were reported (Smith et al. 2011, Li et al. 2013). Barakat et al. (2018), in their study, observed neurodegeneration of hippocampal neurons in mice prenatally treated with DEHP. These authors suggested that the neurodegeneration may be caused by neuronal inflammation and oxidative damage to DNA. This statement was supported by the elevated levels of inflammatory markers and DNA oxidation damage markers in the DEHP mice.

\section{Abnormalities in the amygdala and prefrontal cortex}

The amygdala is involved in the production and recognition of emotions. It reacts to emotionally potent 
stimuli, assigns significance to stimuli, and is involved in the consolidation of stimuli into the memory (Anderson and Phelps 2001, Schultz 2005). The amygdala is functionally connected with the prefrontal cortex and together they have a role in the regulation of goaldirected behavior (Ghashghaei et al. 2007, Ochsner et al. 2012). Moreover, the amygdala and the prefrontal cortex were suggested as being important for social behavior and were implicated in the pathophysiology of ASD (Baron-Cohen et al. 2000, Liu et al. 2020).

When studies aimed to investigate a possible mechanism by which an autistic phenotype might be induced, often, abnormal functional connectivity was observed between the amygdala and cortical regions (Pitskel et al. 2014, Ibrahim et al. 2019, Shen et al. 2016, Gibbard et al. 2018, Sato et al. 2020). The authors suggested that diminished connectivity may play a role in emotion dysregulation (Pitskel et al. 2014, Ibrahim et al. 2019) and the atypicalities in social interaction seen in ASD (Sato et al. 2020). Furthermore, some findings indicate that weaker connectivity between the amygdala and prefrontal cortex is correlated with increased autism severity (Shen et al. 2016, Gibbard et al. 2018). Structural abnormality of the amygdala was observed in autistic brains in a study by Schumann and Amaral (2006). Significantly fewer neurons were counted in the autistic amygdala compared to the control and the authors concluded that the amygdala undergoes atypical development in individuals with autism (Schumann and Amaral 2006). Abnormal cytoarchitecture and disorganization of neurons was observed in prefrontal cortical tissue from children with ASD and the authors speculated that such neuropathological features may probably result from dysregulation of layer formation and layer-specific neuronal differentiation at prenatal developmental stages (Stoner et al. 2014).

The amygdala and medial prefrontal cortex were found to be affected by prenatal and early postnatal phthalate exposure. In a study by DeBartolo et al. (2016), molecular abnormalities in the amygdala were found after prenatal and early postnatal treatment with benzyl butyl phthalate. Levels of methyl CpG binding protein 2, which is important for normal synaptic plasticity and transmission (and its role in the pathology of ASD was recognized (Nagarajan et al. 2006)), were decreased in the amygdala of female rats. The authors suggested that this change may be related to altered learning processes (observed in the study). An environmentally relevant mixture of phthalates was administered in a dose presumably within the range of the estimated daily intake of humans in a study by Kougias et al. (2018a). The mixture caused a reduction in neuron number, synapse number, and size of the medial prefrontal cortex in laboratory rats across both sexes (Kougias et al. 2018a). Results from a study by Komada et al. (2016) suggested that prenatal exposure of mice to DEHP could inhibit neurogenesis, reduce proliferation, and induce cell death of neural stem cells as well as induce abnormal neuronal distribution in the neocortex. On the other hand, in a study by DeBartolo et al. (2016), neurohistological analysis found no adverse changes in neuronal migration and lamination of the neocortex.

\section{Autistic-like traits observed in rodents exposed to phthalates}

ASD is a range of childhood-onset neurodevelopmental disorders. They comprise a broad spectrum of heterogeneous disorders, including autistic disorder, Asperger's disorder, and pervasive developmental disorders not otherwise specified (PDD-NOS). The diagnosis is mostly based on behaviors and ASD is characterized by following core behavioral deficits: persistent deficits in social interaction and social communication (including restricted verbal and nonverbal communication) and restricted repetitive patterns of behavior, interests, or activities. These symptoms are present from early childhood and impair everyday functioning (American Psychiatric Association 2013). Additional associated features are common. A subset of patients with ASD tend to also display anxiety, depression, learning disability, reduction of spatial working memory abilities, hyperactivity, intellectual impairment, motor deficits, language impairment, selfinjury, or disruptive behaviors (O'Brien and Pearson 2004, Steele et al. 2007, American Psychiatric Association 2013).

Evidence from twin and family studies suggest a strong genetic component in the etiology of ASD (Bailey et al. 1995, Luhrs et al. 2017). Despite this, concordance of the behavioral phenotype for autism in monozygotic pairs is not $100 \%$, and the degree of impairment and range of symptoms vary among concordant pairs, and no single gene or common genetic variants have been identified to explain the majority of ASD cases (Bourgeron 2016, Heavner and Smith 2020). This paradox has led to the notion that environmental influences, such as exposure to chemicals and pollutants, are most likely etiologically significant as well (Goodrich et al. 2018). 
Results presented in several human studies suggest that increased prenatal and early postnatal phthalate metabolite concentrations are indeed connected to neurodevelopmental disability (Whyatt et al. 2012, Kim et al. 2018), behavioral problems (Minatoya et al. 2018, Hyland et al. 2019, Jankowska et al. 2019), and autism-like behavior in children (Miodovnik et al. 2011,
Oulhote et al. 2020).

Together with human studies, experimental studies on laboratory animals have also highlighted the possible damage that can be done by prenatal and early postnatal exposure to phthalates; these studies are reviewed below (for more details and suggested pathomechanisms, see Table 1).

Table 1. Behavioral alterations in rodents observed after prenatal and early postnatal phthalate exposure.

\begin{tabular}{|c|c|c|c|c|c|c|c|c|}
\hline \multirow{2}{*}{ Reference } & \multirow{2}{*}{$\begin{array}{c}\text { Administered } \\
\text { phthalates } \\
\text { and dose }\end{array}$} & \multirow{2}{*}{$\begin{array}{c}\text { Days of } \\
\text { administration }\end{array}$} & \multirow{2}{*}{$\begin{array}{l}\text { Model } \\
\text { species }\end{array}$} & \multirow{2}{*}{ Tests } & \multicolumn{3}{|c|}{ Results } & \multirow{2}{*}{$\begin{array}{c}\text { Suggested } \\
\text { pathomechanisms }\end{array}$} \\
\hline & & & & & Both sexes & Males & Females & \\
\hline $\begin{array}{c}\text { Barakat et } \\
\text { al. } 2018\end{array}$ & $\begin{array}{c}\text { DEHP; } 200 \mu \mathrm{g}, \\
500 \mathrm{mg} \text {, or } 750 \\
\mathrm{mg} / \mathrm{kg} / \text { day }\end{array}$ & GD 11 - birth & CD-1 mice & $\begin{array}{c}\text { EPM, } \\
\text { OFT, } \\
\text { Y-maze, } \\
\text { NOR }\end{array}$ & $\begin{array}{l}\text { OFT: } 200 \mu \mathrm{g}, \\
500 \mathrm{mg}, \\
750 \mathrm{mg} / \mathrm{kg} \\
\text { DEHP: fewer } \\
\text { entries to the } \\
\text { central area. } \\
\text { EPM: } \\
750 \mathrm{mg} / \mathrm{kg} \\
\text { DEHP: more } \\
\text { time before } \\
\text { making entries } \\
\text { to the open } \\
\text { arms. Y-maze: } \\
200 \mu \mathrm{g} / \mathrm{kg} \\
\text { DEHP: the } \\
\text { least } \\
\text { alternation } \\
\text { behavior and } \\
\text { the fewest arm } \\
\text { entries. NOR: } \\
500 \text { and } \\
750 \mathrm{mg} / \mathrm{kg} \\
\text { DEHP: less } \\
\text { time exploring } \\
\text { the new } \\
\text { object. }\end{array}$ & & & $\begin{array}{l}\text { Neurodegeneration } \\
\text { of hippocampal } \\
\text { neurons caused by } \\
\text { DEHP. The } \\
\text { neurodegeneration } \\
\text { was probably caused } \\
\text { by the neuronal } \\
\text { inflammation and } \\
\text { oxidative damage to } \\
\text { DNA. }\end{array}$ \\
\hline $\begin{array}{l}\text { Boberg et } \\
\text { al. } 2011\end{array}$ & $\begin{array}{c}\text { DiNP; } 300,600, \\
750 \text { or } 900 \\
\mathrm{mg} / \mathrm{kg} / \text { day }\end{array}$ & GD 7 - PND 17 & Wistar rats & MWM & & $\begin{array}{l}\text { No } \\
\text { significant } \\
\text { difference } \\
\text { found. }\end{array}$ & $\begin{array}{l}900 \mathrm{mg} / \mathrm{kg} \\
\text { DiNP: } \\
\text { reduction in } \\
\text { swim length } \\
\text { and latency to } \\
\text { reach the } \\
\text { platform. }\end{array}$ & \\
\hline $\begin{array}{l}\text { Dai et al. } \\
2015\end{array}$ & $\begin{array}{l}\text { DEHP; } 10,50, \\
\text { or } 200 \\
\mathrm{mg} / \mathrm{kg} / \text { day }\end{array}$ & GD 7 - PND 21 & ICR mice & $\begin{array}{c}\text { OFT, } \\
\text { MWM }\end{array}$ & & $\begin{array}{c}\text { OFT: } \\
200 \mathrm{mg} / \mathrm{kg} \\
\text { DEHP: } \\
\text { increased the } \\
\text { grooming at } \\
\text { age } 6 \text { weeks. } \\
\text { MWM: } 50 \\
\text { and } \\
200 \mathrm{mg} / \mathrm{kg} \\
\text { DEHP: } \\
\text { prolonged the } \\
\text { time of } \\
\text { searching the }\end{array}$ & $\begin{array}{c}\text { OFT: } \\
10 \mathrm{mg} / \mathrm{kg} \\
\text { DEHP: } \\
\text { increased the } \\
\text { grooming at } \\
\text { age } 12 \text { weeks. } \\
10 \text { and } \\
200 \mathrm{mg} / \mathrm{kg} \\
\text { DEHP: } \\
\text { decreased the } \\
\text { frequency of } \\
\text { rearing at age } \\
6 \text { weeks, }\end{array}$ & $\begin{array}{l}\text { Impact on behaviors } \\
\text { was associated with } \\
\text { the inhibition of n- } \\
\text { methyl-D-aspartate } \\
\text { receptor of the } \\
\text { hippocampus. } \\
\text { Authors speculated } \\
\text { that the synaptic } \\
\text { transmission might } \\
\text { have been impaired. }\end{array}$ \\
\hline
\end{tabular}




\begin{tabular}{|c|c|c|c|c|c|c|c|c|}
\hline & & & & & & $\begin{array}{l}\text { hidden } \\
\text { platform in } \\
\text { water maze } \\
\text { and reduced } \\
\text { the time } \\
\text { staying in the } \\
\text { target } \\
\text { quadrant } \\
\text { during a } \\
\text { probe trial of } \\
6 \text {-w-old male } \\
\text { mice. }\end{array}$ & $\begin{array}{l}\text { decreased the } \\
\text { number of } \\
\text { grid crossings } \\
\text { at age } \\
12 \text { weeks. }\end{array}$ & \\
\hline $\begin{array}{l}\text { Hatcher et } \\
\text { al. } 2019\end{array}$ & $\begin{array}{c}\text { DEHP; } 20 \text { or } \\
200 \mu \mathrm{g} / \mathrm{kg} / \text { day, } \\
500 \text { or } 750 \\
\mathrm{mg} / \mathrm{kg} / \text { day }\end{array}$ & GD $10.5-19.5$ & CD-1 mice & EPM & & $\begin{array}{l}\text { F3: No } \\
\text { significant } \\
\text { difference } \\
\text { found. }\end{array}$ & $\begin{array}{c}\text { F3: } \\
750 \mathrm{mg} / \mathrm{kg} \\
\text { DEHP: } \\
\text { increased time } \\
\text { spent in the } \\
\text { open arms. }\end{array}$ & $\begin{array}{l}\text { Authors associated } \\
\text { the transgenerational } \\
\text { effect of DEHP with } \\
\text { epigenetic changes. }\end{array}$ \\
\hline $\begin{array}{l}\text { Hoshi and } \\
\text { Ohtsuka } \\
2009\end{array}$ & $\begin{array}{c}\text { DBP; } 10 \text { or } \\
1000 \mu \mathrm{g} / \mathrm{kg} / \text { day }\end{array}$ & GD 8 - birth & $\begin{array}{l}\text { Sprague- } \\
\text { Dawley } \\
\text { rats }\end{array}$ & $\begin{array}{l}\text { Analysis } \\
\text { of } \\
\text { grooming } \\
\text { behavior }\end{array}$ & & $\begin{array}{l}10 \mu \mathrm{g} / \mathrm{kg} \\
\mathrm{DBP} \text { : lower } \\
\text { frequency of } \\
\text { individual } \\
\text { grooming } \\
\text { bouts. } \\
1.0 \mathrm{mg} / \mathrm{kg} \\
\mathrm{DBP}: \mathrm{no} \\
\text { decrease in } \\
\text { the frequency } \\
\text { of individual } \\
\text { grooming } \\
\text { bouts. }\end{array}$ & & \\
\hline $\begin{array}{l}\text { Kougias et } \\
\text { al. 2018a }\end{array}$ & $\begin{array}{c}\text { DEP, DEHP, } \\
\text { DBP, DiNP, } \\
\text { DiBP, BBP; 0, } \\
\text { 200, or } 1000 \\
\mu \mathrm{g} / \mathrm{kg} / \text { day }\end{array}$ & GD 2 - PND 10 & $\begin{array}{l}\text { Long- } \\
\text { Evans } \\
\text { hooded rats }\end{array}$ & $\begin{array}{l}\text { Attentional } \\
\text { set-shifting } \\
\text { task }\end{array}$ & $\begin{array}{l}\text { Phthalate } \\
\text { mixture: } \\
\text { decreased } \\
\text { cognitive } \\
\text { flexibility } \\
\text { (less correct } \\
\text { responses, } \\
\text { more } \\
\text { perseverative } \\
\text { errors, more } \\
\text { omission } \\
\text { errors). }\end{array}$ & & & $\begin{array}{l}\text { Neuroanatomical } \\
\text { changes of the } \\
\text { medial prefrontal } \\
\text { cortex: reduction in } \\
\text { neuron number, } \\
\text { synapse number, and } \\
\text { the size of the medial } \\
\text { prefrontal cortex. }\end{array}$ \\
\hline $\begin{array}{l}\text { Kougias et } \\
\text { al. } 2018 \mathrm{~b}\end{array}$ & $\begin{array}{c}\text { DEP, DEHP, } \\
\text { DBP, DiNP, } \\
\text { DiBP, BBP; 0, } \\
200, \text { or } \\
1000 \mu \mathrm{g} / \mathrm{kg} / \text { day }\end{array}$ & GD 2 - PND 10 & $\begin{array}{l}\text { Long- } \\
\text { Evans } \\
\text { hooded rats }\end{array}$ & $\begin{array}{c}\text { Social play } \\
\text { behavior }\end{array}$ & & $\begin{array}{c}200 \mathrm{mg} \\
\text { phthalates } / \mathrm{kg}: \\
\text { close to } \\
\text { significant } \\
\text { decrease in } \\
\text { social play. }\end{array}$ & & $\begin{array}{l}\text { Authors speculated } \\
\text { that the anti- } \\
\text { androgenic effect of } \\
\text { phthalates might } \\
\text { have caused } \\
\text { decreased social play } \\
\text { behavior (social play } \\
\text { is affected by } \\
\text { androgens). }\end{array}$ \\
\hline $\begin{array}{l}\text { Lee } e t a l \text {. } \\
2016\end{array}$ & $\begin{array}{c}\text { DEHP; } \\
30 \mathrm{mg} / \mathrm{kg} / \mathrm{day}\end{array}$ & $\begin{array}{c}\text { Pregnancy + } \\
\text { lactation }\end{array}$ & $\begin{array}{c}\text { Wild-type } \\
\text { (WT) mice } \\
\text { with } \\
\text { C57BL/6 } \\
\text { background }\end{array}$ & $\begin{array}{c}\text { OFT, } \\
\text { EPM, SIT } \\
\text { and MWM }\end{array}$ & $\begin{array}{l}\text { SIT: DEHP: } \\
\text { decreased } \\
\text { social } \\
\text { preference to a } \\
\text { stranger } \\
\text { mouse. }\end{array}$ & & & $\begin{array}{l}\text { Maternal DEHP } \\
\text { exposure might have } \\
\text { affected the prenatal } \\
\text { differentiation of } \\
\text { neurons in offspring } \\
\text { and result in } \\
\text { impaired ability for } \\
\text { social interaction. }\end{array}$ \\
\hline
\end{tabular}




\begin{tabular}{|c|c|c|c|c|c|c|c|c|}
\hline $\begin{array}{l}\text { Li et al. } \\
2013\end{array}$ & $\begin{array}{c}\text { DBP; } \\
500 \mathrm{mg} / \mathrm{kg} / \text { day }\end{array}$ & GD 6 - PND 21 & $\begin{array}{l}\text { Sprague } \\
\text { Dawley } \\
\text { rats }\end{array}$ & MWM & $\begin{array}{c}\text { DBP: } \\
\text { decreased } \\
\text { numbers of } \\
\text { platform } \\
\text { crossings. }\end{array}$ & $\begin{array}{l}\text { DBP: } \\
\text { increased } \\
\text { latency to } \\
\text { escape. }\end{array}$ & $\begin{array}{l}\text { No significant } \\
\text { difference } \\
\text { found. }\end{array}$ & $\begin{array}{l}\text { DBP exposure might } \\
\text { have induced } \\
\text { synaptic loss and } \\
\text { synaptic function } \\
\text { failure, leading to } \\
\text { cognitive } \\
\text { impairment. }\end{array}$ \\
\hline $\begin{array}{l}\text { Lin et al. } \\
2015\end{array}$ & $\begin{array}{l}\text { DEHP; } 10 \text { or } \\
750 \mathrm{mg} / \mathrm{kg} / \text { day }\end{array}$ & GD $12-21$ & $\begin{array}{l}\text { Sprague } \\
\text { Dawley } \\
\text { rats }\end{array}$ & MWM & $\begin{array}{l}10 \text { and } \\
750 \mathrm{mg} / \mathrm{kg} \\
\text { DEHP: } \\
\text { prolonged } \\
\text { escape } \\
\text { latencies, } \\
\text { fewer times } \\
\text { across a } \\
\text { platform. } \\
750 \mathrm{mg} / \mathrm{kg} \\
\text { DEHP: less } \\
\text { search time in } \\
\text { quadrant with } \\
\text { the platform. }\end{array}$ & & & $\begin{array}{l}\text { DEHP altered the } \\
\text { expression of genes } \\
\text { critical for neuron } \\
\text { proliferation. } \\
\text { Authors suggested } \\
\text { that this might have } \\
\text { partially contributed } \\
\text { to the deficit of } \\
\text { cognitive function. }\end{array}$ \\
\hline $\begin{array}{l}\text { Quinnies et } \\
\text { al. } 2015\end{array}$ & $\begin{array}{l}\text { DEHP; } 150 \text { or } \\
200 \mathrm{mg} / \mathrm{kg} / \text { day }\end{array}$ & GD $7-14$ & $\begin{array}{l}\mathrm{C} 57 \mathrm{BL} / 6 \mathrm{~J} \\
\text { mice }\end{array}$ & SIT, EPM & & $\begin{array}{l}\text { F3: SIT: } \\
200 \mathrm{mg} / \mathrm{kg} \\
\text { DEHP: more } \\
\text { time spent in } \\
\text { digging and } \\
\text { less time } \\
\text { spent in self- } \\
\text { grooming. }\end{array}$ & & $\begin{array}{l}\text { Authors associated } \\
\text { the transgenerational } \\
\text { effect of DEHP with } \\
\text { epigenetic changes. }\end{array}$ \\
\hline $\begin{array}{l}\text { Quinnies et } \\
\text { al. } 2017\end{array}$ & $\begin{array}{c}\text { DEHP; } 5,40 \text {, or } \\
400 \mu \mathrm{g} / \mathrm{kg} / \text { day }\end{array}$ & GD 0.5 - PND 10 & $\begin{array}{l}\mathrm{C} 57 \mathrm{BL} / 6 \mathrm{~J} \\
\text { mice }\end{array}$ & SIT, EPM & $\begin{array}{l}\text { F1: SIT: } 40, \\
400 \mu \mathrm{g} / \mathrm{kg} \\
\text { DEHP: less } \\
\text { sitting alone. } \\
40 \mu \mathrm{g} / \mathrm{kg} \\
\text { DEHP: sniffed } \\
\text { their test } \\
\text { partner more } \\
\text { frequently. } \\
400 \mu \mathrm{g} / \mathrm{kg} \\
\text { DEHP: more } \\
\text { exploratory } \\
\text { behaviors. } \\
\text { EPM: } 5, \\
40 \mu \mathrm{g} / \mathrm{kg} \\
\text { DEHP: more } \\
\text { time in the } \\
\text { closed arms. } \\
\text { F3: less } \\
\text { exploratory } \\
\text { behaviors. }\end{array}$ & $\begin{array}{l}\text { F1: SIT: 40, } \\
400 \mu \mathrm{g} / \mathrm{kg} \\
\text { DEHP: less } \\
\text { sitting side- } \\
\text { by-side. } \\
\text { F3: } \\
400 \mu \mathrm{g} / \mathrm{kg} \\
\text { DEHP: more } \\
\text { sitting side- } \\
\text { by-side, more } \\
\text { sitting alone. }\end{array}$ & $\begin{array}{l}\text { F3: } 400 \mu \mathrm{g} / \mathrm{kg} \\
\text { DEHP: more } \\
\text { sitting alone. }\end{array}$ & $\begin{array}{l}\text { Authors suggested } \\
\text { possible impact of } \\
\text { DEHP on } \\
\text { neurodevelopment. } \\
\text { Anxiety-like } \\
\text { behavior might have } \\
\text { been caused by the } \\
\text { hypothalamic- } \\
\text { pituitary-adrenal axis } \\
\text { disturbance. The } \\
\text { transgenerational } \\
\text { effect of DEHP was } \\
\text { associated with } \\
\text { epigenetic changes. }\end{array}$ \\
\hline $\begin{array}{l}\text { Sellinger et } \\
\text { al. } 2020\end{array}$ & $\begin{array}{c}\text { DEP, DEHP, } \\
\text { DBP, DiNP, } \\
\text { DiBP, BBP; 0, } \\
\text { 200, or } 1000 \\
\mu \mathrm{g} / \mathrm{kg} / \text { day }\end{array}$ & GD 2 - PND 10 & $\begin{array}{c}\text { Long- } \\
\text { Evans } \\
\text { hooded rats }\end{array}$ & EPM & & $\begin{array}{l}\text { No } \\
\text { significant } \\
\text { difference } \\
\text { found. }\end{array}$ & $\begin{array}{l}\text { No significant } \\
\text { difference } \\
\text { found. }\end{array}$ & \\
\hline $\begin{array}{l}\text { Xu et al. } \\
2015\end{array}$ & $\begin{array}{l}\text { DEHP; } 10,50, \\
\text { or } 200 \\
\mathrm{mg} / \mathrm{kg} / \text { day }\end{array}$ & GD 7 - PND 21 & ICR mice & $\begin{array}{c}\text { OFT, } \\
\text { Dark/light } \\
\text { transition } \\
\text { task, The } \\
\text { mirrored } \\
\text { maze task, }\end{array}$ & & $\begin{array}{c}\text { OFT: } \\
200 \mathrm{mg} / \mathrm{kg} \\
\text { DEHP } \\
\text { significantly } \\
\text { increased the } \\
\text { frequency of }\end{array}$ & $\begin{array}{l}\text { OFT: } 10 \text { and } \\
200 \mathrm{mg} / \mathrm{kg} \\
\text { DEHP: } \\
\text { decreased the } \\
\text { frequency of } \\
\text { rearing at age }\end{array}$ & $\begin{array}{c}\text { Authors suggested } \\
\text { that anxiety- and } \\
\text { depression-like } \\
\text { behaviors were due } \\
\text { to the } \\
\text { downregulation of }\end{array}$ \\
\hline
\end{tabular}




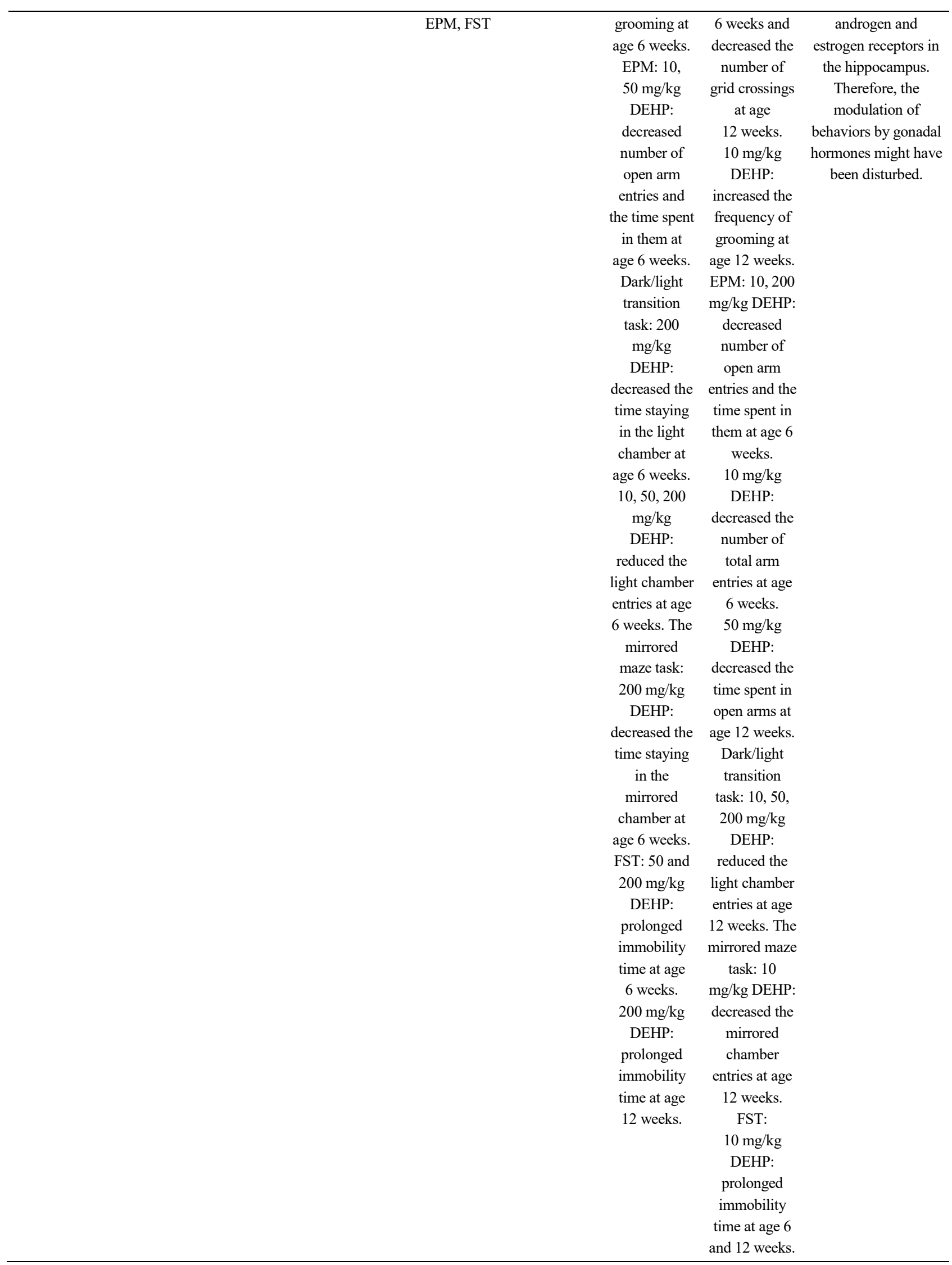

Abbreviations: BBP - benzyl butyl phthalate, DBP - di-n-butyl phthalate, DEHP - di(2-ethylhexyl)phthalate, DEP - diethyl phthalate, DiBP - di-isobutyl phthalate, DiNP - diisononyl phthalate, EPM - Elevated plus maze, F1 - one generation removed from phthalate exposure, F3 - three generations removed from phthalate exposure, FST - Forced swim test, GD - gestational day, MWM - Morris water maze, NOR - Novel object recognition test, OFT - Open field test, PND - postnatal day, SIT - Social interaction test. 


\section{Impact on social behavior}

Negative impact of prenatal and early postnatal exposure to phthalates on social behavior was recorded in several studies. Exposure to DEHP during pregnancy and lactation caused a decrease in social preference to a stranger mouse in young adult offspring mice (Lee et al. 2016). A negative impact on social behavior was also observed in a study by Kougias et al. (2018b), where prenatal and early postnatal exposure to a mixture of phthalates caused a decrease in social play in periadolescent rats. In a study by Quinnies et al. (2017), juvenile mice exposed to DEHP prenatally and early postnatally displayed fewer socially investigative behaviors.

In some studies, the transgenerational effect of phthalates was assessed to investigate if the impact of these substances persists through multiple generations, but results are inconclusive. Transgenerational DEHP exposure had no effect on social behavior in juvenile mice (only males were used) but they spent more time in nonsocial behavior (Quinnies et al. 2015). In another study, however, F3 male mice from a DEHP lineage were more interactive and spent less time in independent behavior (no difference was found in females) (Quinnies et al. 2017).

Anxiety-like behavior, depression-like behavior, and emotional deficits

Anxiety-like, as well as depression-like behavior (so-called because they resemble anxiety and depression behaviors in humans, though in rodents they might represent something else (Lezak et al. 2017)) were observed after prenatal phthalate exposure. In a study by Quinnies et al. (2017), mice had increased anxiety-like behavior after they were exposed to DEHP prenatally and early postnatally. These findings are in accordance with the results of another, earlier study (Xu et al. 2015), in which anxiety-like behavior was increased in pubertal mice regardless of sex, and in adults, when females were affected. On the other hand, Lee et al. (2016) and Sellinger et al. (2020) did not observe anxiety-like behavior in rodents after prenatal and early postnatal exposure to phthalates. Depression-like behavior was found to be induced in pubertal and adult mice after prenatal and postnatal phthalate exposure (Xu et al. 2015).

In transgenerational studies, no effect of DEHP lineage on anxiety-like behavior in F3 juvenile male or female mice was observed (Quinnies et al. 2015, Quinnies et al. 2017). In the most recent study, transgene- rational DEHP exposure had an anxiolytic effect in adult mice from the F3 generation, but only in females. The authors suggest that this behavioral alteration indicates maladaptive anxiety-like behavior or hyperactivity rather than a therapeutic effect (Hatcher et al. 2019).

Adverse effects on emotional stability in a novel environment after phthalate exposure during prenatal development were indicated in a study by Hoshi and Ohtsuka (2009) and in a study by Dai et al. (2015). Adult male rats (Hoshi and Ohtsuka 2009) and pubertal male and adult female mice (Dai et al. 2015) exhibited changes in grooming behavior after they were placed in a test cage.

Impact on cognition, learning, and memory

Impairment of learning and spatial memory was observed in adult male rats after prenatal exposure to DEHP (Lin et al. 2015) and in male pubertal mice after prenatal and lactational exposure to this chemical (Dai et al. 2015). Learning and spatial memory was also impaired in young rats (postnatal day 22) exposed to DBP from gestational day six to postnatal day 21 ( $\mathrm{Li}$ et al. 2013). On the other hand, prenatal and postnatal exposure to diisononyl phthalate (DiNP) resulted in better performance in tests for spatial learning in young adult rats, but this improvement was seen only in females. No changes were seen in males. As phthalates are endocrine disruptors, the authors suggested that hormonal imbalance during the critical periods of brain development could alter the default female outcome in a more masculine direction (Boberg et al. 2011). Lee et al. (2016) did not observe any changes in spatial learning and memory in young adult mice exposed to DEHP during lactation and gestation. In a more recent study, spatial and recognition memory were assessed in adult male mice after prenatal exposure to DEHP (Barakat et al. 2018). This exposure correlated with reduced spatial memory function and impaired recognition memory.

Kougias et al. (2018a) tested cognitive flexibility of adult rats in an attentional set-shifting task after prenatal and early postnatal phthalate exposure. Cognitive flexibility can be explained as the ability to learn a new strategy while inhibiting the execution of a strategy that was previously correct (Ragozzino et al. 1999). Rats were trained to enter a target arm of a testing apparatus using a specific cue based on color to obtain a food reward. The next day they were trained to enter a target arm using a cue based on texture. Whether a rat performed a correct choice or an error across the trials was analyzed. Vehicleexposed rats performed better and made fewer errors than phthalate mixture-exposed rats in the testing apparatus. 
On the initial discrimination training, however, phthalateexposed rats did not perform worse. These authors suggest that prenatal and early postnatal exposure to an environmentally relevant mixture of phthalates resulted in a long-term deficit in cognitive flexibility rather than general learning ability (Kougias et al. 2018a).

\section{Rodents prenatally and early postnatally affected by phthalates - An animal model of autism?}

As we have already stated, ASD is a disorder whose etiology is not yet known. In order to elucidate the fundamental causes behind this disorder and to develop reliable diagnostic methods and medication or some other methods to control the symptoms, many animal models are currently being used. Animal models play a substantial role in understanding this disease by enabling investigators to carry out experiments that would be impractical or ethically prohibited with humans. Available models include those with genetic, physiological, and behavioral relevance to an autistic phenotype. Such a phenotype can be induced by generating genetic mutations, brain lesions, or by administrating chemical substances (e.g. valproic acid). The matter of available animal models for ASD was reviewed in detail elsewhere (Olexová et al. 2012, Ergaz et al. 2016, Möhrle et al. 2020). Here, we would like to discuss the autistic phenotype observed in rodents after prenatal exposure to phthalates and whether it would be possible to use prenatal exposure to these chemicals in the development of a new animal model of autism.

The defining criteria for ASD are behavioral, therefore, to evaluate external manifestations of this disorder in animal models (i.e. autistic-like traits), behavioral test techniques are used (Crawley 2012). Usually, social interaction tests are used to evaluate social behavior. In this test, social responses and time spent in social interactions are measured in pairs of animals (Quinnies et al. 2017). Anxiety-like behavior is often determined by the elevated plus maze test where time spent in open and closed arms of a maze is measured. Less time spent in open arms and more time spent in closed arms is indicative of anxiety-like behavior (Quinnies et al. 2017). Depression is evaluated by the forced swim test, where an animal is forced to swim in a container from which it cannot escape. Increased immobility then demonstrates depression-like behavior (Xu et al. 2015). For analyzing motor activity and repetitive behaviors, the open field test is used. An animal is allowed to move freely in an arena with walls and its motor behavior is monitored (Barakat et al. 2018). Memory, learning, and cognitive ability are often measured by the Morris water maze (an animal has to swim to find the hidden platform and then across trials, it is expected to remember its position (Lin et al. 2015)) or the novel object recognition test (one of two known objects is replaced by a novel object and the amount of time taken to explore the novel object provides an index of recognition memory (Barakat et al. 2018). There is no doubt that prenatal exposure to phthalates affects the behavior of rodents. However, it yet remains unanswered whether the altered behavior can really be considered as an autistic phenotype. In animal models of autism, the behavior that is most frequently studied is social behavior. Social behavior was affected, and sociability was decreased after prenatal and early postnatal exposure to phthalates in a number of studies mentioned above (Lee et al. 2016, Quinnies et al. 2017, Kougias et al. 2018b). The occurrence of motor stereotypes and repetitive behaviors is also being examined when phenotyping mouse and rat models of autism. Regarding motor stereotypes, we were unable to find any studies addressing this parameter. Self-grooming is sometimes considered a repetitive behavior (Kalueff et al. 2016). Hoshi and Ohtsuka (2009) and Dai et al. (2015) investigated self-grooming after prenatal and postnatal phthalate exposure, however, it was to estimate the emotional state of the animals rather than to assess repetitive behavior. Moreover, results from these studies were contradictory, and no definitive conclusion can be drawn at this point. Behaviors relevant to associated symptoms of ASD are also being investigated. Anxietylike and depression-like behaviors were observed in several animal studies assessing exposure to phthalates during prenatal and postnatal phthalate exposure (Xu et al. 2015, Quinnies et al. 2017). Cognitive abilities and cognitive flexibility were negatively affected by prenatal and early postnatal phthalate exposure (Lin et al. 2015, Kougias et al. 2018a). Hyperactivity might also be related to phthalate exposure, but results are inconclusive. Ishido et al. (2005) administered phthalates to male rats in early postnatal development and observed an increased spontaneous motor activity during the nocturnal phase later in life. On the other hand, motor activity levels of exposed male and female rodent offspring were not affected in studies by Boberg et al. (2011) and Lee et al. (2016), moreover, Dai et al. (2015) found that horizontal and vertical motor activity was decreased in female mice after prenatal and postnatal exposure to phthalates. 
From the studies we have reviewed so far, we can say that this possible animal model of autism has only one out of three types of validity that are sought from animal models (Bourin et al. 2007). First is face validity, where the model is phenotypically similar and implies that the response observed in the animal model should be identical to the behavioral and physiological responses observed in humans. So far, it seems that in both humans and rodents, the behavioral and physiological impact of prenatal phthalate exposure is mostly the same. Second is construct validity, requiring that the etiology of the behavioral and biological factors underlying the disorder may be similar in animals and humans. The cause for abnormalities in reproductive tract development observed in rodents and humans after prenatal phthalate exposure seems to be a reduction of steroidogenesis and therefore, reduced function of steroids (Hannas et al. 2012). However, it is not yet clear how phthalates induce an autistic-like phenotype in rodents and humans. Reduced function of testosterone may play a role, especially in prenatal development when this hormone is essential for brain development (NegriCesi et al. 2004). Changes in brain structure and function described above in section 2 had a negative impact on cognition, learning, memory (Li et al. 2013, Dai et al. 2015, DeBartolo et al. 2016, Kougias et al. 2018a), and motor activity (Ishido et al. 2005). Moreover, interference with normal synaptogenesis and connectivity as well as morphological changes in different brain areas were suggested as potential causes for ASD (Ha et al. 2015, Guang et al. 2018). Therefore, changes caused by prenatal and early postnatal phthalate exposure in the development of the brain could indeed be responsible for an autistic phenotype, but more studies would be necessary to clearly address the construct validity of this model. Predictive validity (entails that the model should be sensitive to clinically effective pharmacological agents) is even harder to assess. One can say that, for now at least, it is impossible as there is no standard effective pharmacological treatment for ASD yet (Reichow et al. 2018).

One final point we would like to consider is that for ASD to manifest, it seems that genetic abnormalities and certain environmental conditions must be present. This means that we need not only understand the consequences of the genetic and environmental perturbations as such, we also need to examine their interactions. If, for example, a model based on genetic manipulations were to be combined with the environmental one and tested and described thoroughly, such a model could meet the criteria for all three types of validity, and this could lead to the development of one universal model of autism which could reflect this condition accurately and reliably.

\section{Conclusions}

In this review, we presented the existing body of evidence regarding the effect of prenatal and early postnatal exposure to phthalates on neurodevelopment and behavior and highlighted the possibility of phthalates having a role in the emergence of ASD. Autistic-like traits observed in animal studies, as well as the prospect of using developmental exposure to phthalates as a new animal model of autism, were also discussed.

Prenatal and early postnatal exposure to phthalates in rodents resulted in abnormalities in brain development and behavior observable in individuals with ASD. This could indicate the possibility of creating a new animal model of autism. However, it should be noted that not all tests used in reviewed studies are unanimously accepted for the measurement of ASD-like behaviors. Considering the information on validity, more studies would be necessary to determine if the "phthalate animal model of autism" has high translational value. Still, considering the results of reviewed studies, we believe that this matter should be further investigated, and more importantly, developmental phthalate exposure might be considered as an ASD risk factor.

\section{Conflict of Interest}

There is no conflict of interest.

\section{Acknowledgements}

This work was supported by grants VEGA 2/0166/16, VEGA $2 / 0154 / 20$ and UK/73/2018.

\section{References}

AMERICAN PSYCHIATRIC ASSOCIATION: Diagnostic and statistical manual of mental disorders, Fifth edition.

$\begin{array}{lllllll}\text { American } \quad \text { Psychiatric } & \text { Association, } & \text { Arlington, } & \text { VA, } & 2013, & \text { pp } & \text { 50-59. } \\ \text { https://doi.org/10.1176/appi.books.9780890425596 } & & & & \end{array}$


ANDERSON AK, PHELPS EA: Lesions of the human amygdala impair enhanced perception of emotionally salient events. Nature 411: 305-309, 2001. https://doi.org/10.1038/35077083

ANDERSON EB, GROSSRUBATSCHER I, FRANK L: Dynamic hippocampal circuits support learning- and memoryguided behaviors. Cold Spring Harb Symp Quant Biol 79: 51-58, 2014. https://doi.org/10.1101/sqb.2014.79.024760

BAILEY A, LE COUTEUR A, GOTTESMAN I, BOLTON P, SIMONOFF E, YUZDA E, RUTTER M: Autism as a strongly genetic disorder: evidence from a British twin study. Psychol Med 25: 63-77, 1995. https://doi.org/10.1017/S0033291700028099

BARAKAT R, LIN PC, PARK CJ, BEST-POPESCU C, BAKRY HH, ABOSALEM ME, ABDELALEEM NM, FLAWS JA, KO C: Prenatal exposure to DEHP induces neuronal degeneration and neurobehavioral abnormalities in adult male mice. Toxicol Sci 164: 439-452, 2018. https://doi.org/10.1093/toxsci/kfy103

BARON-COHEN S, RING HA, BULLMORE ET, WHEELWRIGHT S, ASHWIN C, WILLIAMS SC: The amygdala theory of autism. Neurosci Biobehav Rev 24: 355-364, 2000. https://doi.org/10.1016/S0149-7634(00)00011-7

BARTOS M, ELGUETA C: Functional characteristics of parvalbumin- and cholecystokinin-expressing basket cells. J Physiol 590: 669-681, 2012. https://doi.org/10.1113/jphysiol.2011.226175

BLYSTONE CR, KISSLING GE, BISHOP JB, CHAPIN RE, WOLFE GW, FOSTER PM: Determination of the di-(2ethylhexyl) phthalate NOAEL for reproductive development in the rat: importance of the retention of extra animals to adulthood. Toxicol Sci 116: 640-646, 2010. https://doi.org/10.1093/toxsci/kfq147

BOBERG J, CHRISTIANSEN S, AXELSTAD M, KLEDAL TS, VINGGAARD AM, DALGAARD M, NELLEMANN C, HASS U: Reproductive and behavioral effects of diisononyl phthalate (DINP) in perinatally exposed rats. Reprod Toxicol 31: 200-209, 2011. https://doi.org/10.1016/j.reprotox.2010.11.001

BOURGERON T: Current knowledge on the genetics of autism and propositions for future research. C R Biol 339: 300-307, 2016. https://doi.org/10.1016/j.crvi.2016.05.004

BOURIN M, PETIT-DEMOULIÈRE B, DHONNCHADHA BN, HASCÖET M: Animal models of anxiety in mice. Fundam Clin Pharmacol 21: 567-574, 2007. https://doi.org/10.1111/j.1472-8206.2007.00526.x

CHEN J, YU S, FU Y, LI X: Synaptic proteins and receptors defects in autism spectrum disorders. Front Cell Neurosci 8: 276, 2014. https://doi.org/10.3389/fncel.2014.00276

COHEN NJ: Navigating life. Hippocampus 25: 704-708, 2015. https://doi.org/10.1002/hipo.22443

COLBORN T: Neurodevelopment and endocrine disruption. Environ Health Perspect 112: 944-949, 2004. https://doi.org/10.1289/ehp.6601

CRAWLEY JN: Translational animal models of autism and neurodevelopmental disorders. Dialogues Clin Neurosci 14: 293-305, 2012. https://doi.org/10.31887/DCNS.2012.14.3/jcrawley

DAI Y, YANG Y, XU X, HU Y: Effects of uterine and lactational exposure to di-(2-ethylhexyl) phthalate on spatial memory and NMDA receptor of hippocampus in mice. Horm Behav 71: 41-48, 2015. https://doi.org/10.1016/j.yhbeh.2015.03.008

DEBARTOLO D, JAYATILAKA S, YAN SIU N, ROSE M, RAMOS RL, BETZ AJ: Perinatal exposure to benzyl butyl phthalate induces alterations in neuronal development/maturation protein expression, estrogen responses, and fear conditioning in rodents. Behav Pharmacol 27: 77-82, 2016. https://doi.org/10.1097/FBP.0000000000000190

DENG X, GU L, SUI N, GUO J, LIANG J: Parvalbumin interneuron in the ventral hippocampus functions as a discriminator in social memory. Proc Natl Acad Sci U S A 116: 16583-16592, 2019. https://doi.org/10.1073/pnas.1819133116

DIAMANTI-KANDARAKIS E, BOURGUIGNON J-P, GIUDICE LC, HAUSER R, PRINS GS, SOTO AM, ZOELLER RT, GORE AC: Endocrine-disrupting chemicals: an endocrine society scientific statement. Endocr Rev 30: 293-342, 2009. https://doi.org/10.1210/er.2009-0002

DONG D, ZIELKE HR, YEH D, YANG P: Cellular stress and apoptosis contribute to the pathogenesis of autism spectrum disorder. Autism Res 11: 1076-1090, 2018. https://doi.org/10.1002/aur.1966

ERGAZ Z, WEINSTEIN-FUDIM L, ORNOY A: Genetic and non-genetic animal models for autism spectrum disorders (ASD). Reprod Toxicol 64: 116-140, 2016. https://doi.org/10.1016/j.reprotox.2016.04.024

FACTOR-LITVAK P, INSEL B, CALAFAT AM, LIU X, PERERA F, RAUH VA, WHYATT RM: Persistent associations between maternal prenatal exposure to phthalates on child IQ at age 7 years. PLoS One 9: e114003, 2014. https://doi.org/10.1371/journal.pone.0114003 
FREDERIKSEN H, SKAKKEBAEK NE, ANDERSSON AM: Metabolism of phthalates in humans. Mol Nutr Food Res 51: 899-911, 2007. https://doi.org/10.1002/mnfr.200600243

GHASHGHAEI HT, HILGETAG CC, BARBAS H: Sequence of information processing for emotions based on the anatomic dialogue between prefrontal cortex and amygdala. Neuroimage 34: 905-923, 2007. https://doi.org/10.1016/j.neuroimage.2006.09.046

GIBBARD CR, REN J, SKUSE DH, CLAYDEN JD, CLARK CA: Structural connectivity of the amygdala in young adults with autism spectrum disorder. Hum Brain Mapp 39: 1270-1282, 2018. https://doi.org/10.1002/hbm.23915

GITLER D, TAKAGISHI Y, FENG J, REN Y, RODRIGUIZ RM, WETSEL WC, GREENGARD P, AUGUSTINE GJ: Different presynaptic roles of synapsins at excitatory and inhibitory synapses. J Neurosci 24: 11368-11380, 2004. https://doi.org/10.1523/JNEUROSCI.3795-04.2004

GOODRICH AJ, VOLK HE, TANCREDI DJ, MCCONNELL R, LURMANN FW, HANSEN RL, SCHMIDT RJ: Joint effects of prenatal air pollutant exposure and maternal folic acid supplementation on risk of autism spectrum disorder. Autism Res 11: 69-80, 2018. https://doi.org/10.1002/aur.1885

GRANDJEAN P, LANDRIGAN PJ: Developmental neurotoxicity of industrial chemicals. Lancet 368: 2167-2178, 2006. https://doi.org/10.1016/S0140-6736(06)69665-7

GUANG S, PANG N, DENG X, YANG L, HE F, WU L, CHEN C, YIN F, PENG J: Synaptopathology involved in autism spectrum disorder. Front Cell Neurosci 12: 470, 2018. https://doi.org/10.3389/fncel.2018.00470

HA S, SOHN IJ, KIM N, SIM HJ, CHEON KA: Characteristics of brains in autism spectrum disorder: structure, function and connectivity across the lifespan. Exp Neurobiol 24: 273-284, 2015. https://doi.org/10.5607/en.2015.24.4.273

HANNAS BR, LAMBRIGHT CS, FURR J, EVANS N, FOSTER PM, GRAY EL, WILSON VS: Genomic biomarkers of phthalate-induced male reproductive developmental toxicity: a targeted RT-PCR array approach for defining relative potency. Toxicol Sci 125: 544-557, 2012. https://doi.org/10.1093/toxsci/kfr315

HATCHER KM, WILLING J, CHIANG C, RATTAN S, FLAWS JA, MAHONEY MM: Exposure to di-(2-ethylhexyl) phthalate transgenerationally alters anxiety-like behavior and amygdala gene expression in adult male and female mice. Physiol Behav 207: 7-14, 2019. https://doi.org/10.1016/j.physbeh.2019.04.018

HAUSER R, MEEKER JD, PARK S, SILVA MJ, CALAFAT AM: Temporal variability of urinary phthalate metabolite levels in men of reproductive age. Environ Health Perspect 112: 1734-1740, 2004. https://doi.org/10.1289/ehp.7212

HEAVNER WE, SMITH SEP: Resolving the synaptic versus developmental dichotomy of autism risk genes. Trends Neurosci 43: 227-241, 2020. https://doi.org/10.1016/j.tins.2020.01.009

HITTI FL, SIEGELBAUM SA: The hippocampal CA2 region is essential for social memory. Nature 508: 88-92, 2014. https://doi.org/10.1038/nature13028

HOPPIN JA, BROCK JW, DAVIS BJ, BAIRD DD: Reproducibility of urinary phthalate metabolites in first morning urine samples. Environ Health Perspect 110: 515-518, 2002. https://doi.org/10.1289/ehp.02110515

HOSHI H, OHTSUKA T: Adult rats exposed to low-doses of di-n-butyl phthalate during gestation exhibit decreased grooming behavior. Bull Environ Contam Toxicol 83: 62-66, 2009. https://doi.org/10.1007/s00128-009-9729-1

HSIEH MH, BREYER BN, EISENBERG ML, BASKIN LS: Associations among hypospadias, cryptorchidism, anogenital distance, and endocrine disruption. Curr Urol Rep 9: 137-142, 2008. https://doi.org/10.1007/s11934-008-0025-0

HU D, WANG YX, CHEN WJ, ZHANG Y, LI HH, XIONG L, ZHU HP, CHEN HY, PENG SX, WAN ZH, ZHANG Y, DU YK: Associations of phthalates exposure with attention deficits hyperactivity disorder: A case-control study among Chinese children. Environ Pollut 229: 375-385, 2017. https://doi.org/10.1016/i.envpol.2017.05.089

HYLAND C, MORA AM, KOGUT K, CALAFAT AM, HARLEY K, DEARDORFF J, HOLLAND N, ESKENAZI B, SAGIV SK: Prenatal exposure to phthalates and neurodevelopment in the CHAMACOS cohort. Environ Health Perspect 127: 107010, 2019. https://doi.org/10.1289/EHP5165

IBRAHIM K, EILBOTT JA, VENTOLA P, HE G, PELPHREY KA, MCCARTHY G, SUKHODOLSKY DG: Reduced amygdala-prefrontal functional connectivity in children with autism spectrum disorder and co-occurring disruptive behavior. Biol Psychiatry Cogn Neurosci Neuroimaging 4: 1031-1041, 2019. https://doi.org/10.1016/j.bpsc.2019.01.009

ISHIDO M, MORITA M, OKA S, MASUO Y: Alteration of gene expression of G protein-coupled receptors in endocrine disruptors-caused hyperactive rats. Regul Pept 126: 145-153, 2005. https://doi.org/10.1016/j.regpep.2004.08.035 
JANKOWSKA A, POLAŃSKA K, HANKE W, WESOŁOWSKA E, LIGOCKA D, WASZKOWSKA M, STAŃCZAK A, TARTAGLIONE AM, MIRABELLA F, CHIAROTTI F, GARÍ M, CALAMANDREI G: Prenatal and early postnatal phthalate exposure and child neurodevelopment at age of 7 years - Polish Mother and Child Cohort. Environ Res 177: 108626, 2019. https://doi.org/10.1016/j.envres.2019.108626

KALUEFF AV, STEWART AM, SONG C, BERRIDGE KC, GRAYBIEL AM, FENTRESS JC: Neurobiology of rodent self-grooming and its value for translational neuroscience. Nat Rev Neurosci 17: 45-59, 2016. https://doi.org/10.1038/nrn.2015.8

KIM S, EOM S, KIM HJ, LEE JJ, CHOI G, CHOI S, KIM S, KIM SY, CHO G, KIM YD, SUH E, KIM SK, KIM S, KIM GH, MOON HB, PARK J, KIM S, CHOI K, EUN SH: Association between maternal exposure to major phthalates, heavy metals, and persistent organic pollutants, and the neurodevelopmental performances of their children at 1 to 2years of age- CHECK cohort study. Sci Total Environ 624: 377-384, 2018. https://doi.org/10.1016/j.scitotenv.2017.12.058

KLEIN D, KESSLER W, SEMDER B, PÜTZ C, LICHTMANNEGGER J, OTTER R, FILSER JG: Di-(2-propylheptyl) phthalate (DPHP) and its metabolites in blood of rats upon single oral administration of DPHP. Toxicol Lett 259: 80-86, 2016. https://doi.org/10.1016/j.toxlet.2016.07.025

KOCH HM, BOLT HM, PREUSS R, ANGERER J: New metabolites of di(2-ethylhexyl) phthalate (DEHP) in human urine and serum after single oral doses of deuterium-labelled DEHP. Arch Toxicol 79: 367-376, 2005. https://doi.org/10.1007/s00204-004-0642-4

KOLATOROVA L, VITKU J, VAVROUS A, HAMPL R, ADAMCOVA K, SIMKOVA M, PARIZEK A, STARKA L, DUSKOVA M: Phthalate metabolites in maternal and cord plasma and their relations to other selected endocrine disruptors and steroids. Physiol Res 67 (Suppl 3): S473-S487, 2018. https://doi.org/10.33549/physiolres.933962

KOMADA M, GENDAI Y, KAGAWA N, NAGAO T: Prenatal exposure to di(2-ethylhexyl) phthalate impairs development of the mouse neocortex. Toxicol Lett 259: 69-79, 2016. https://doi.org/10.1016/j.toxlet.2016.07.019

KOUGIAS DG, CORTES LR, MOODY L, RHOADS S, PAN YX, JURASKA JM: Effects of perinatal exposure to phthalates and a high-fat diet on maternal behavior and pup development and social play. Endocrinology 159: 1088-1105, 2018b. https://doi.org/10.1210/en.2017-03047

KOUGIAS DG, SELLINGER EP, WILLING J, JURASKA JM: Perinatal exposure to an environmentally relevant mixture of phthalates results in a lower number of neurons and synapses in the medial prefrontal cortex and decreased cognitive flexibility in adult male and female rats. J Neurosci 38: 6864-6872, 2018a. https://doi.org/10.1523/JNEUROSCI.0607-18.2018

LEE E, LEE J, KIM E: Excitation/inhibition imbalance in animal models of autism spectrum disorders. Biol Psychiatry 81: 838-847, 2017. https://doi.org/10.1016/j.biopsych.2016.05.011

LEE KI, CHIANG CW, LIN HC, ZHAO JF, LI CT, SHYUE SK, LEE TS: Maternal exposure to di-(2-ethylhexyl) phthalate exposure deregulates blood pressure, adiposity, cholesterol metabolism and social interaction in mouse offspring. Arch Toxicol 90: 1211-1224, 2016. https://doi.org/10.1007/s00204-015-1539-0

LEZAK KR, MISSIG G, CARLEZON WA JR: Behavioral methods to study anxiety in rodents. Dialogues Clin Neurosci 19: 181-191, 2017. https://doi.org/10.31887/DCNS.2017.19.2/wcarlezon

LI XJ, JIANG L, CHEN L, CHEN HS, LI X: Neurotoxicity of dibutyl phthalate in brain development following perinatal exposure: a study in rats. Environ Toxicol Pharmacol 36: 392-402, 2013. https://doi.org/10.1016/j.etap.2013.05.001

LIN H, YUAN K, LI L, LIU S, LI S, HU G, LIAN QQ, GE RS: In utero exposure to diethylhexyl phthalate affects rat brain development: a behavioral and genomic approach. Int J Environ Res Public Health 12: 13696-13710, 2015. https://doi.org/10.3390/ijerph121113696

LINTELMANN J, KATAYAMA A, KURIHARA N, SHORE L, WENZEL A: Endocrine disruptors in the environment (IUPAC technical report). Pure Appl Chem 75: 631-681, 2003. https://doi.org/10.1351/pac200375050631

LIU X, BAUTISTA J, LIU E, ZIKOPOULOS B: Imbalance of laminar-specific excitatory and inhibitory circuits of the orbitofrontal cortex in autism. Mol Autism 11: 83, 2020. https://doi.org/10.1186/s13229-020-00390-X

LUHRS K, WARD T, HUDAC CM, GERDTS J, STESSMAN HAF, EICHLER EE, BERNIER RA: Associations between familial rates of psychiatric disorders and de novo genetic mutations in autism. Autism Res Treat 2017: 9371964, 2017. https://doi.org/10.1155/2017/9371964 
MARTINO-ANDRADE AJ, CHAHOUD I: Reproductive toxicity of phthalate esters. Mol Nutr Food Res 54: 148-157, 2010. https://doi.org/10.1002/mnfr.200800312

MESSERLIAN C, BELLINGER D, MÍNGUEZ-ALARCÓN L, ROMANO ME, FORD JB, WILLIAMS PL, CALAFAT AM, HAUSER R, BRAUN JM: Paternal and maternal preconception urinary phthalate metabolite concentrations and child behavior. Environ Res 158: 720-728, 2017. https://doi.org/10.1016/j.envres.2017.07.032

MINATOYA M, ITOH S, YAMAZAKI K, ARAKI A, MIYASHITA C, TAMURA N, YAMAMOTO J, ONODA Y, OGASAWARA K, MATSUMURA T, KISHI R: Prenatal exposure to bisphenol A and phthalates and behavioral problems in children at preschool age: The Hokkaido Study on Environment and Children's Health. Environ Health Prev Med 23: 43, 2018. https://doi.org/10.1186/s12199-018-0732-1

MIODOVNIK A, ENGEL SM, ZHU C, YE X, SOORYA LV, SILVA MJ, CALAFAT AM, WOLFF MS: Endocrine disruptors and childhood social impairment. Neurotoxicology 32: 261-267, 2011. https://doi.org/10.1016/j.neuro.2010.12.009

MÖHRLE D, FERNÁNDEZ M, PEÑAGARIKANO O, FRICK A, ALLMAN B, SCHMID S: What we can learn from a genetic rodent model about autism. Neurosci Biobehav Rev 109: 29-53, 2020. https://doi.org/10.1016/j.neubiorev.2019.12.015

MÜlLEROVÁ D, BOUCHALOVÁ V, MATĚJKOVÁ D, KOVÁŘOVÁ K, SVAČINA Š, VRBíK K, PAVLOUŠKOVÁ J, DVOŘÁKOVÁ J, MÜLLER L: Phthalates exposure indicators determined by urinary phthalate metabolites in healthy non-obese Czech adults: FANTOM study. Food Addit Contam Part A Chem Anal Control Expo Risk Assess 33: 1817-1825, 2016. https://doi.org/10.1080/19440049.2016.1241898

NAGARAJAN RP, HOGART AR, GWYE Y, MARTIN MR, LASALLE JM: Reduced MeCP2 expression is frequent in autism frontal cortex and correlates with aberrant MECP2 promoter methylation. Epigenetics 1: e1-e11, 2006. https://doi.org/10.4161/epi.1.4.3514

NEGRI-CESI P, COLCIAGO A, CELOTTI F, MOTTA M: Sexual differentiation of the brain: role of testosterone and its active metabolites. J Endocrinol Invest 27: 120-127, 2004.

O'BRIEN G, PEARSON J: Autism and learning disability. Autism 8: 125-140, 2004. https://doi.org/10.1177/1362361304042718

OCHSNER KN, SILVERS JA, BUHLE JT: Functional imaging studies of emotion regulation: a synthetic review and evolving model of the cognitive control of emotion. Ann N Y Acad Sci 1251: E1-E24, 2012. https://doi.org/10.1111/j.1749-6632.2012.06751.x

O'KEEFE J, DOSTROVSKY J: The hippocampus as a spatial map. Preliminary evidence from unit activity in the freely-moving rat. Brain Res 34: 171-175, 1971. https://doi.org/10.1016/0006-8993(71)90358-1

OLEXOVÁ L, TALAROVIČOVÁ A, LEWIS-EVANS B, BORBÉLYOVÁ V, KRŠKOVÁ L: Animal models of autism with a particular focus on the neural basis of changes in social behaviour: an update article. Neurosci Res 74: 184-194, 2012. https://doi.org/10.1016/j.neures.2012.10.004

OULHOTE Y, LANPHEAR B, BRAUN JM, WEBSTER GM, ARBUCKLE TE, ETZEL T, FORGET-DUBOIS N, SEGUIN JR, BOUCHARD MF, MACFARLANE A, OUELLET E, FRASER W, MUCKLE G: Gestational exposures to phthalates and folic acid, and autistic traits in Canadian children. Environ Health Perspect 128: 27004, 2020. https://doi.org/10.1289/EHP5621

PISKOROWSKI RA, NASRALLAH K, DIAMANTOPOULOU A, MUKAI J, HASSAN SI, SIEGELBAUM SA, GOGOS JA, CHEVALEYRE V: Age-dependent specific changes in area CA2 of the hippocampus and social memory deficit in a mouse model of the 22q11.2 deletion syndrome. Neuron 89: 163-176, 2016. https://doi.org/10.1016/j.neuron.2015.11.036

PITSKEL NB, BOLLING DZ, KAISER MD, PELPHREY KA, CROWLEY MJ: Neural systems for cognitive reappraisal in children and adolescents with autism spectrum disorder. Dev Cogn Neurosci 10: 117-128, 2014. https://doi.org/10.1016/j.den.2014.08.007

QIN X, FENG J, CAO C, WU H, LOH YP, CHENG Y: Association of peripheral blood levels of brain-derived neurotrophic factor with autism spectrum disorder in children: a systematic review and meta-analysis. JAMA Pediatr 170: 1079-1086, 2016. https://doi.org/10.1001/jamapediatrics.2016.1626

QUINNIES KM, DOYLE TJ, KIM KH, RISSMAN EF: Transgenerational effects of di-(2-ethylhexyl) phthalate (DEHP) on stress hormones and behavior. Endocrinology 156: 3077-3083, 2015. https://doi.org/10.1210/EN.2015-1326 
QUINNIES KM, HARRIS EP, SNYDER RW, SUMNER SS, RISSMAN EF: Direct and transgenerational effects of low doses of perinatal di-(2-ethylhexyl) phthalate (DEHP) on social behaviors in mice. PLoS One 12: e0171977, 2017. https://doi.org/10.1371/journal.pone.0171977

RAGOZZINO ME, DETRICK S, KESNER RP: Involvement of the prelimbic-infralimbic areas of the rodent prefrontal cortex in behavioral flexibility for place and response learning. J Neurosci 19: 4585-4594, 1999. https://doi.org/10.1523/JNEUROSCI.19-11-04585.1999

REICHOW B, HUME K, BARTON EE, BOYD BA: Early intensive behavioral intervention (EIBI) for young children with autism spectrum disorders (ASD). Cochrane Database Syst Rev 5: CD009260, 2018. https://doi.org/10.1002/14651858.CD009260.pub3

REPOUSKOU A, PANAGIOTIDOU E, PANAGOPOULOU L, BISTING PL, TUCK AR, SJÖDIN MOD, LINDBERG J, BOZAS E, RÜEGG J, GENNINGS C, BORNEHAG CG, DAMDIMOPOULOU P, STAMATAKIS A, KITRAKI E: Gestational exposure to an epidemiologically defined mixture of phthalates leads to gonadal dysfunction in mouse offspring of both sexes. Sci Rep 9: 6424, 2019. https://doi.org/10.1038/s41598-019-42377-6

RHEE J, PARK K, KIM KC, SHIN CY, CHUNG C: Impaired hippocampal synaptic plasticity and enhanced excitatory transmission in a novel animal model of autism spectrum disorders with telomerase reverse transcriptase overexpression. Mol Cells 41: 486-494, 2018. https://doi.org/10.14348/molcells.2018.0145

RING M, DERWENT CLT, GAIGG SB, BOWLER DM: Structural learning difficulties implicate altered hippocampal functioning in adults with autism spectrum disorder. J Abnorm Psychol 126: 793-804, 2017. https://doi.org/10.1037/abn0000277

SATO W, UONO S, KOCHIYAMA T: Neurocognitive mechanisms underlying social atypicalities in autism: weak amygdala's emotional modulation hypothesis. Front Psychiatry 11: 864, 2020. https://doi.org/10.3389/fpsyt.2020.00864

SCENIAK MP, FEDDER KN, WANG Q, DROUBI S, BABCOCK K, PATWARDHAN S, WRIGHT-ZORNES J, PHAM L, SABO SL: An autism-associated mutation in GluN2B prevents NMDA receptor trafficking and interferes with dendrite growth. J Cell Sci 132: jcs232892, 2019. https://doi.org/10.1242/jcs.232892

SCHETTLER T: Human exposure to phthalates via consumer products. Int J Androl 29: 134-139, 2006. https://doi.org/10.1111/j.1365-2605.2005.00567.x

SCHULTZ RT: Developmental deficits in social perception in autism: the role of the amygdala and fusiform face area. Int J Dev Neurosci 23: 125-141, 2005. https://doi.org/10.1016/j.ijdevneu.2004.12.012

SCHUMANN CM, AMARAL DG: Stereological analysis of amygdala neuron number in autism. J Neurosci 26: 7674-7679, 2006. https://doi.org/10.1523/JNEUROSCI.1285-06.2006

SELLINGER EP, KOUGIAS DG, DRZEWIECKI CM, JURASKA JM: Behavioral effects in adult rats exposed to low doses of a phthalate mixture during the perinatal or adolescent period. Neurotoxicol Teratol 79: 106886, 2020. https://doi.org/10.1016/j.ntt.2020.106886

SHEN MD, LI DD, KEOWN CL, LEE A, JOHNSON RT, ANGKUSTSIRI K, ROGERS SJ, MÜLLER RA, AMARAL DG, NORDAHL CW: Functional connectivity of the amygdala is disrupted in preschool-aged children with autism spectrum disorder. J Am Acad Child Adolesc Psychiatry 55: 817-824, 2016. https://doi.org/10.1016/j.jaac.2016.05.020

SMITH CA, HOLAHAN MR: Reduced hippocampal dendritic spine density and BDNF expression following acute postnatal exposure to di(2-ethylhexyl) phthalate in male Long Evans rats. PLoS One 9: e109522, 2014. https://doi.org/10.1371/journal.pone.0109522

SMITH CA, MACDONALD A, HOLAHAN MR: Acute postnatal exposure to di(2-ethylhexyl) phthalate adversely impacts hippocampal development in the male rat. Neuroscience 193: 100-108, 2011. https://doi.org/10.1016/j.neuroscience.2011.06.082

STEELE SD, MINSHEW NJ, LUNA B, SWEENEY JA: Spatial working memory deficits in autism. J Autism Dev Disord 37: 605-612, 2007. https://doi.org/10.1007/s10803-006-0202-2

STONER R, CHOW ML, BOYLE MP, SUNKIN SM, MOUTON PR, ROY S, WYNSHAW-BORIS A, COLAMARINO SA, LEIN ES, COURCHESNE E: Patches of disorganization in the neocortex of children with autism. N Engl J Med 370: 1209-1219, 2014. https://doi.org/10.1056/NEJMoa1307491 
SWAN SH, MAIN KM, LIU F, STEWART SL, KRUSE RL, CALAFAT AM, MAO CS, REDMON JB, TERNAND CL, SULLIVAN S, TEAGUE JL; STUDY FOR FUTURE FAMILIES RESEARCH TEAM: Decrease in anogenital distance among male infants with prenatal phthalate exposure. Environ Health Perspect 113: 1056-1061, 2005. https://doi.org/10.1289/ehp.8100

TSAI YA, TSAI MS, HOU JW, LIN CL, CHEN CY, CHANG CH, LIAO KW, WANG SL, CHEN BH, WU MT, HSIEH CJ, CHEN ML; TMICS GROUP: Evidence of high di(2-ethylhexyl) phthalate (DEHP) exposure due to tainted food intake in Taiwanese pregnant women and the health effects on birth outcomes. Sci Total Environ 618: 635-644, 2018. https://doi.org/10.1016/j.scitotenv.2017.07.175

WENG TI, CHEN MH, LIEN GW, CHEN PS, LIN JCC, FANG CC, CHEN PC: Effects of gender on the association of urinary phthalate metabolites with thyroid hormones in children: A Prospective Cohort Study in Taiwan. Int J Environ Res Public Health 14: 123, 2017. https://doi.org/10.3390/ijerph14020123

WHYATT RM, LIU X, RAUH VA, CALAFAT AM, JUST AC, HOEPNER L, DIAZ D, QUINN J, ADIBI J, PERERA FP, FACTOR-LITVAK P: Maternal prenatal urinary phthalate metabolite concentrations and child mental, psychomotor and behavioral development at 3years of age. Environ Health Perspect 120: 290-295, 2012. https://doi.org/10.1289/ehp.1103705

XU X, YANG Y, WANG R, WANG Y, RUAN Q, LU Y: Perinatal exposure to di-(2-ethylhexyl) phthalate affects anxiety- and depression-like behaviors in mice. Chemosphere 124: 22-31, 2015. https://doi.org/10.1016/j.chemosphere.2014.10.056

ZAMKOWSKA D, KARWACKA A, JUREWICZ J, RADWAN M: Environmental exposure to non-persistent endocrine disrupting chemicals and semen quality: An overview of the current epidemiological evidence. Int J Occup Med Environ Health 31: 377-414, 2018. https://doi.org/10.13075/ijomeh.1896.01195

ZHANG YW, GAO H, MAO LJ, TAO XY, GE X, HUANG K, ZHU P, HAO JH, WANG QN, XU YY, JIN ZX, SHENG J, XU YQ, YAN SQ, TAO XG, TAO FB: Effects of the phthalate exposure during three gestation periods on birth weight and their gender differences: A birth cohort study in China. Sci Total Environ 613-614: 1573-1578, 2018. https://doi.org/10.1016/j.scitotenv.2017.08.319 